\title{
Aceite de trementina modificado: un inhibidor de enzimas de- toxificadoras en dos cepas de Aedes aegypti
}

\author{
Maureen Leyva ${ }^{1}$, Leidys French ${ }^{2}$, María del Carmen Marquetti ${ }^{1}$, Domingo Montada ${ }^{1}$, Mayda Cas- \\ tex ${ }^{1}$, Olinka Tiomno ${ }^{3}$, Juan E Tacoronte ${ }^{4}$ \\ ${ }^{1}$ Instituto de Medicina Tropical "Pedro Kour'”, La Habana, Cuba, ${ }^{2}$ Centro de Investigaciones Químicas (CIQ) Univer- \\ sidad Autónoma del Estado de Morelos, México, ${ }^{3}$ Centro de Investigaciones Químicas (CIQ) La Habana, Cuba, \\ ${ }^{4}$ SENESCYT, Universidad Central del Ecuador, Quito, Ecuador
}

\section{RESUMEN}

Introducción. Los principales mecanismos de resistencia a los insecticidas desarrollados por los insectos incluyen una serie de enzimas detoxificadoras. Se conoce que estos mecanismos pudieran afectar la efectividad en la actividad insecticida de algunos aceites esenciales. Estudiar la implicación de las enzimas detoxificadoras es importante cuando se pretende recomendar un aceite esencial para el control vectorial.

Objetivo. Determinar la actividad de las enzimas de acción metabólica en dos cepas de Ae. aegypti (susceptible y resistente a insecticidas) al ser expuestas a sus respectivas $\mathrm{CL}_{90}$ de un aceite de trementina obtenido de Pinus tropicalis y modificado por fotoisomerización.

Resultados. Los valores de actividad de las $\beta$-esterasa y MFO oxidasa no variaron en la cepa susceptible; sin embargo, en la cepa resistente ocurrió una disminución de los valores de estas actividades. La actividad de las GST mostró inhibición en la cepa susceptible, mientras que, para la cepa resistente se encontró un ligero incremento.

Conclusiones. En este trabajo se pudo demostrar que las enzimas de acción metabólica evaluadas no constituyen un mecanismo de resistencia en las larvas que sobrevivieron a la exposición del aceite de trementina modificado. Este resultado, unido a los efectos biológicos de este aceite, así como su actividad ovicida, larvicida, pupicida y teratogénica, permite recomendar su uso como un método alternativo para el control de Aedes aegypti.

Palabras clave: Aedes aegypti, GST, esterasas, aceite esencial

\section{ABSTRACT \\ Modified turpentine oil as an inhibitor of detoxifying enzymes in two strains of Aedes aegypti mosquitoes}

Introduction. The main mechanisms of insecticide resistance developed by insects include a series of detoxifying enzymes. It is known that these mechanisms may affect the effectiveness of the insecticidal activity of some essential oils. To study the implication of detoxifying enzymes is very important when trying to recommend an essential oil for vector control.

Objective. To determine the activity of enzymes with metabolic action in strains of Ae. aegypti exposed and unexposed to modified turpentine oil .

Autor para correspondencia: Maureen Leyva Silva, Instituto de Medicina Tropical “Pedro Kour'”" (IPK) Autopista Novia del Mediodía Km 6 ² Apartado 601, La Lisa, La Habana, Cuba . E-mail: maureen@ipk.sld.cu

Recibido: el 22 de agosto de 2014. Aceptado para publicación: el 2 de diciembre de 2014

Este documento está disponible en http://www.revbiomed.uady.mx/pdf/rb152613.pdf 


\section{Leyva-Silva et al.}

Results. The values of the activity from $\beta$-esterase and MFO oxidase did not change in the selected $\mathrm{CL}_{90}$ susceptible strain. However, there was a decrease in the values of these activities in the selected resistance strain. GST activity was inhibited in the susceptible strain and increased slightly for the resistant strain. This study demonstrated that the enzymes of metabolic action do not constitute a mechanism of resistance in the larvae that survived the exposure to the modified turpentine oil.

Conclusion. This result, combined with the biological effects of this oil as ovicide, larvicide, teratogenic and pupicidal, allows recommending its use as a biological alternative method for controlling Aedes aegypti.

Key Words: Aedes aegypti, GTS, esterases, essential oils

\section{INTRODUCCIÓN}

Aedes (Stegomyia) aegypti (Diptera: Culicidae) constituye el vector primario de dengue en las Américas (1). Se estima que aproximadamente, $40 \%$ de la población mundial está en riesgo de contraer el virus y entre 50 a 100 millones de personas son infectados anualmente $(2,3)$. Si bien es cierto que el control químico es la opción más utilizada para reducir la transmisión de esta virosis, debido a que no se cuenta con un candidato vacunal efectivo (4), es posible utilizar otras alternativas de control con menos limitaciones ambientales.

Las plantas, en su proceso evolutivo, han desarrollado mecanismos de defensa contra el daño ocasionado por plagas (5). Estas propiedades fueron empleadas desde tiempos inmemoriales por el hombre para evitar las molestias principalmente causadas por insectos (6). Sin embargo, con el advenimiento del uso de los insecticidas sintéticos la utilización de las plantas para el control de plagas disminuyó considerablemente. Una de las consecuencias de la aplicación ininterrumpida de los insecticidas ha sido el desarrollo de mecanismos de resistencia en los insectos (4, 6-10). Esta problemática ha conllevado a retomar el uso de controladores biológicos alternativos como las plantas, pues son una fuente promisoria en la búsqueda de compuestos con actividad insecticida (11-18).

Cuba, posee una extensa y variada flora, de la cual existen especies de plantas biosintetizadoras de sustancias con actividad plaguicida (19). El potencial insecticida de la inmensa mayoría no se ha estudiado a profundidad, salvo algunas evaluaciones realizadas en plagas denla agricultura y de la salud pública (20-24).

En estudios previos se comprobó la actividad larvicida e inhibidora del desarrollo en una cepa de Ae. aegypti resistente a insecticidas, de un aceite de trementina obtenido de la destilación de la resina de Pinus tropicalis y modificado por fotoisomerización (25). Sin embargo, este tipo de actividad biológica, se obtuvo en dicha cepa con un ligero incremento de la dosis subletal, infiriendo que la resistencia a insecticidas sintéticos encontrada y un posible incremento de la actividad de enzimas detoxificadoras, juegan un papel en este resultado.

Debido a que los principales mecanismos de resistencia a los insecticidas desarrollados por los insectos incluyen las carboxilesterasas (EST), glutatión-S-transferasas (GST) y citocromo P450 monooxigenasas (MFO) (26-28), nuestro objetivo fue determinar la actividad de estas enzimas de acción metabólica en dos cepas de Ae. aegypti, una susceptible y otra resistente, expuestas a una $\mathrm{CL}_{90}$ del aceite de trementina modificado (ATM).

\section{MATERIALES Y MÉTODOS}

Cepas de $A$ e. aegypti utilizadas en el estudio. Cepa Rockefeller. Cepa de referencia de laboratorio susceptible a insecticidas, suministrada por el Centro para el Control y la Prevención de Enfermedades (CDC), San Juan, Puerto Rico, 1996.

Cepa San Miguel del Padrón 2011. Cepa colectada en estadios de larva y pupa en el año 
Inhibición de enzimas detoxificadoras en Aedes aegypti

2011, durante una etapa intensiva de control vectorial en el municipio San Miguel del Padrón, situado en La Habana, Cuba.

Bioensayos de susceptibilidad a insecticidas en larvas. La susceptibilidad y/o resistencia al insecticida organofosforado temefos (insecticida utilizado para el control larval en Cuba por el Programa Nacional de Control de Aedes aegypti y Aedes albopictus), fue evaluada, al igual que los insecticidas lambdacialotrina, deltametrina, bendiocarb y malatión, a través de los bioensayos de susceptibilidad para larvas (29). Se utilizaron larvas de tercer estadío tardío o cuarto estadío temprano de las dos cepas de mosquitos. Se aplicaron cinco o más concentraciones del insecticida con acetona, como diluyente. Se emplearon cuatro réplicas y un control por cada concentración que causaron mortalidades entre 2 y 98 \%. La mortalidad se leyó a las 24 horas después de aplicados los insecticidas. Las concentraciones letales se calcularon usando Probit en SPSS versión 11 para Windows. Se calculó el Factor de Resistencia $\left(\mathrm{FR}_{50}, \mathrm{FR}_{99}\right)$, dividiendo el valor de $\mathrm{CL}_{50}, \mathrm{CL}_{99}$ de la cepa San Miguel 2011entre los valores obtenidos con la cepa susceptible Rockefeller.

Se utilizó como criterio de susceptibilidad las siguientes relaciones: $\mathrm{FR}_{50}<5$ Susceptible, $\mathrm{FR}_{50} 5-10$ Resistencia moderada, $\mathrm{FR}_{50}>10$ Resistente (30). $\mathrm{FR}_{99}<2$ Susceptible $\mathrm{FR}_{99} \geq 2$ resistente (31).

Selección con ATM en larvas de Aedes aegypti, cepas San Miguel del Padrón 2011 y cepa Rockefeller. Larvas de Aedes aegypti de la cepa Rockefeller y de la cepa San Miguel del Padrón 2011 fueron expuestas a diferentes dosis de ATM, posteriormente, se calculó la $\mathrm{CL}_{90}$ para cada cepa (0.0043\% y $0.011 \%$, respectivamente) utilizando Probit en SPSS versión 11 para Windows (25). Se utilizaron como control larvas de ambas cepas que no fueron expuestas al aceite y a las que solamente se aplicó $1 \mathrm{ml}$ de etanol. La mortalidad se calculó a las 24 horas; las larvas sobrevivientes se lavaron con abundante agua destilada y fueron colocadas, posteriormente, en un volumen de $500 \mathrm{ml}$ de agua por 24 horas más.

Preparación de la muestra. Para cada ensayo de actividad enzimática se seleccionó una muestra aleatoria de 200 individuos; larvas no expuestas o control y larvas expuestas, pero sobrevivientes de cada cepa. Se colocaron, individualmente, en placas de 96 pocillos para la realización de las pruebas bioquímicas. Se maceraron en $50 \mu \mathrm{L}$ de tampón fosfato $0,01 \mathrm{~mol} / \mathrm{L}, \mathrm{pH}$ 7,5 con el empleo de un homogenizador de placas, sobre una bolsa de hielo. Se completó a un volumen de $300 \mu \mathrm{L}$ de tampón fosfato.

Citocromo P450 mono-oxigenasas. Para la actividad de las enzimas citocromo P450 monooxigenasas se utilizó la metodología descrita por Brogdon et al, (1998)(32) y modificada por French et al, 2013(33). Se utilizaron $80 \mu \mathrm{L}$ de macerado, $200 \mu \mathrm{L}$ de sustrato $3,3^{\prime}, 5,5^{\prime}$ - tetrametilbenzidina (TMBZ) a una concentración de $12 \mathrm{mmol} / \mathrm{L}$, y 25 $\mu \mathrm{L}$ de $\mathrm{H}_{2} \mathrm{O}_{2}(3 \%)$. Se utilizó como control negativo una mezcla del tampón fosfato, TMBZ y $\mathrm{H}_{2} \mathrm{O}_{2}$. La reacción se dejó transcurrir durante 10 min. La absorbancia se leyó a $620 \mathrm{~nm}$, a $25^{\circ} \mathrm{C}$, en un espectrofotómetro cinético (Versa Max, EE.UU).

Glutatión-S-transferasas. Para la actividad de las enzimas Glutatión-s-transferasas se utilizó la metodología descrita por Rodríguez et al, 2001(34). De cada uno de las muestras se tomó 20 $\mu \mathrm{L}$ y se añadieron a una placa de microtitulación de 96 pocillos. Seguidamente se añadieron 200 $\mu \mathrm{L}$ del sustrato [mezcla: $1 \mathrm{~mL}$ de 1-cloro-2,4dinitrobenzeno ( $50 \mathrm{mmol} / \mathrm{L}$ ) diluido en metanol y $20 \mathrm{~mL}$ de glutation reducido $(20 \mathrm{mmol} / \mathrm{L})$ diluido en tampón fosfato]. La reacción se dejó transcurrir durante $3 \mathrm{~min}$. La absorbancia se leyó a 340nm, a $25^{\circ} \mathrm{C}$, en un espectrofotómetro cinético (Versa Max, EE.UU).

a y $\boldsymbol{\beta}$ carboxilesterasas. Para la actividad de 


\section{Leyva-Silva et al.}

las enzimas $\alpha$ y $\beta$ carboxilesterasas se utilizó la metodología descrita por Rodríguez et al, 2001(34). De cada uno de las muestras maceradas se tomó $20 \mu \mathrm{L}$ y se añadieron a una placa de microtitulación de 96 pocillos. Seguidamente se añadieron $200 \mu \mathrm{L}$ de $\alpha$ o $\beta$ naftil acetato (diluido 1:100 a partir de una solución madre de $\alpha$ o $\beta$-naftil acetato $70 \mathrm{mmol} / \mathrm{L}$ ). Se dejó transcurrir la reacción durante $10 \mathrm{~min}$. Posteriormente, se añadieron 40 $\mu \mathrm{L}$ del colorante Fast Blue B Salt $(0,03 \mathrm{~g}$ en 10 $\mathrm{mL}$ de la mezcla $\mathrm{H} 2 \mathrm{O}$ y SDS $5 \%$, 3:7 v/v). La absorbancia se calculó a $570 \mathrm{~nm}$, a $25^{\circ} \mathrm{C}$, en un espectrofotómetro cinético (Versa Max, EE.UU).

Análisis estadístico . Se analizó la normalidad de los datos mediante la prueba Kolmogorov-Smirnov y Shapiro-Wilk. La diferencia estadísticamente significativa de los valores de actividad enzimática en los grupos en estudio, se determinó mediante un test de Mann Whitney, utilizando el software Statistica versión 7.

\section{RESULTADOS}

En el Cuadro 1, se muestra los estudios de susceptibilidad a insecticidas de las 2 cepas en estudio. Se muestran los valores de $\mathrm{CL}_{50}$, $\mathrm{CL}_{99}$ y el $\mathrm{FR}_{50} \mathrm{FR}_{99}$, obtenidos para temefos, lambdacialotrina, deltametrina, malatión y bendiocarb, en ambas cepas.

La cepa San Miguel del Padrón 2011, resultó altamente resistente a los 4 insecticidas utilizados, lo que se evidencia en los factores de resistencia $\left(\mathrm{FR}_{50}>10 \mathrm{x} ; \mathrm{FR}_{99} \geq 2\right)$, según criterio (30-31). El FR ${ }_{50}$ calculado para temefos fue de 4.8x lo cual seria un indicador de susceptibilidad; sin embargo, el $\mathrm{FR}_{99}$ muestra un valor superior a 2. Esta diferencia de criterio pudiera considerarse como un inicio de resistencia moderada de esta cepa frente a temefos, lo que está en concordancia con otros estudios realizados en cepas colectadas en La Habana.

Al estudiar las enzimas implicadas en la resistencia a diferentes insecticidas (Figuras 1 y 2), en las dos cepas, luego de haber sido expuestas

\section{Cuadro 1}

Valores de concentración letal $\left(\mathrm{CL}_{50}, \mathbf{C L}_{99}\right)$ y factor de resistencia $\left(\mathrm{FR}_{50}, \mathrm{FR}_{99}\right)$ de las cepas San Miguel del Padrón 2011 y Rockefeller frente a 4 insecticidas y ATM

\begin{tabular}{|c|c|c|c|c|c|}
\hline $\begin{array}{l}\text { Insecticidas } \\
\text { testados }\end{array}$ & $\begin{array}{c}\text { Rockefeller } \\
\text { CL }_{50}(\%) /(\mathrm{LC})\end{array}$ & $\begin{array}{c}\text { San Miguel del Padrón } \\
2011 \\
\text { CLso }(\%) /(\mathrm{LC})\end{array}$ & $\begin{array}{c}\text { Rockefeller } \\
\text { CL } 9(\%) /(L C)\end{array}$ & $\begin{array}{c}\text { San Miguel del Padrón } \\
2011 \\
\text { CL } 9(\%) /(\mathrm{LC})\end{array}$ & FRsa/FRog \\
\hline Lambdacialotrina & $\begin{array}{c}0.0000014 \\
(0.0000010-0.0000017)\end{array}$ & $\begin{array}{c}0.000047 \\
(0.000035-0.000056)\end{array}$ & $\begin{array}{c}0.0000020 \\
(0.0000018-0.0000022)\end{array}$ & $\begin{array}{c}0.0000961 \\
(0.000094-0.000098)\end{array}$ & $33 / 48$ \\
\hline Deltametrina & $\begin{array}{c}0.0000030 \\
(0.0000010-0.0000050)\end{array}$ & $\begin{array}{c}0.000089 \\
(0.000078-0.00010)\end{array}$ & $\begin{array}{c}0.0000051 \\
(0.0000047-0.0000054)\end{array}$ & $\begin{array}{c}0.000481 \\
(0.00046-0.00051)\end{array}$ & $29 / 96$ \\
\hline Malatión & $\begin{array}{c}0.0000032 \\
(0.0000014-0.0000050)\end{array}$ & $\begin{array}{c}0.000036 \\
(0.000030-0.000048)\end{array}$ & $\begin{array}{c}0.0000085 \\
(0.0000081-0.0000088)\end{array}$ & $\begin{array}{c}0.000111 \\
(0.000107-0.000116)\end{array}$ & $11 / 12.9$ \\
\hline Bendiocarb & $\begin{array}{c}0.0000011 \\
(0.0000089-0.000018)\end{array}$ & $\begin{array}{c}0.0000193 \\
(0.000067-0.00021)\end{array}$ & $\begin{array}{c}0.0000039 \\
(0.0000036-0.0000042)\end{array}$ & $\begin{array}{c}0.000036 \\
(0.000032-0.000038)\end{array}$ & $17 / 9.2$ \\
\hline Temefos & $\begin{array}{c}0.00000535 \\
(0.000052-0.000058)\end{array}$ & $\begin{array}{c}0.000024 \\
(0.00020-0.00029)\end{array}$ & $\begin{array}{c}0.0000162 \\
(0.0000160-0.0000165)\end{array}$ & $\begin{array}{c}0.000098 \\
(0.00096-0.00102)\end{array}$ & $4.8 / 6.1$ \\
\hline $\mathrm{ATM}^{*}$ & $\begin{array}{c}0.0023 \\
(0.0022-0.0025)\end{array}$ & $\begin{array}{c}0.0057 \\
(0.0052-0.0060)\end{array}$ & $\begin{array}{c}0.0055 \\
(0.0047-0.0069)\end{array}$ & $\begin{array}{c}0.0155 \\
(0.0124-0.023)\end{array}$ & $2.4 / 2.8$ \\
\hline
\end{tabular}

LC: Limite confiabilidad de las concentraciones letales calculadas mediante Probit en SPSS versión 11 para Windows

* Estos valores fueron obtenidos con anterioridad en Leyva et al., 2013

\section{Revista Biomédica}


Inhibición de enzimas detoxificadoras en Aedes aegypti
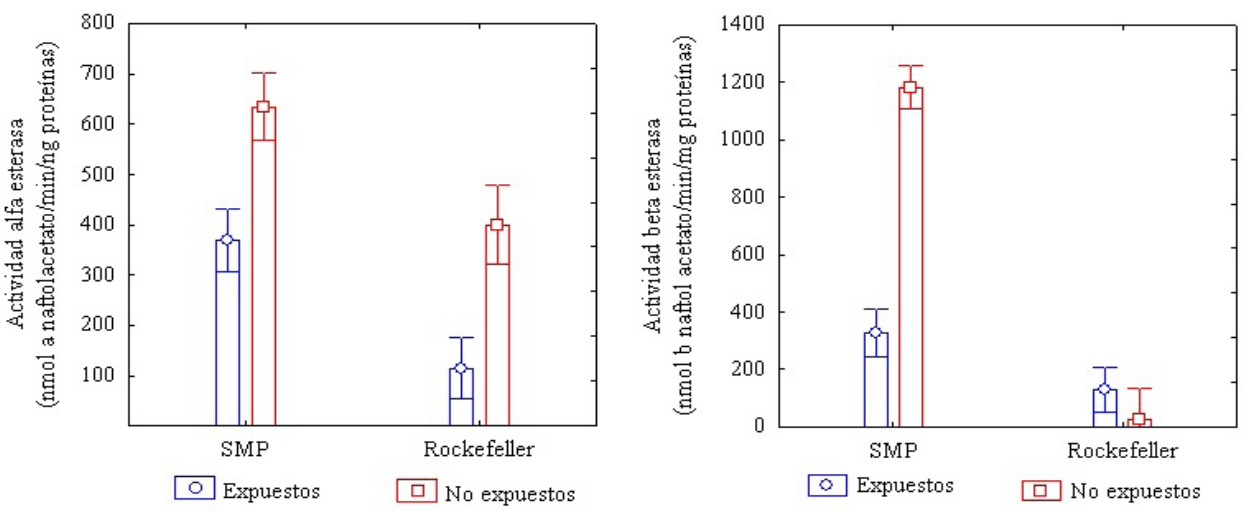

Figura 1. Actividad $\alpha$ esterasa (A) y $\beta$ esterasa (B) determinada para cada cepa en el estadio de larva. Se realizó un test Mann Whitney (A: SMP (U=1404; $p=0,0000001)$; Rockefeller ( $\mathrm{U}=1002 ; \mathrm{p}=0.0000001)$; B: SMP $(\mathrm{U}=396$; $\mathrm{p}=0$, 0000001); Rockefeller $(\mathrm{U}=125 ; \mathrm{p}=0.0000001)$ utilizando el programa estadístico STATISTICA 7. Las barras representan los intervalos de confianza.
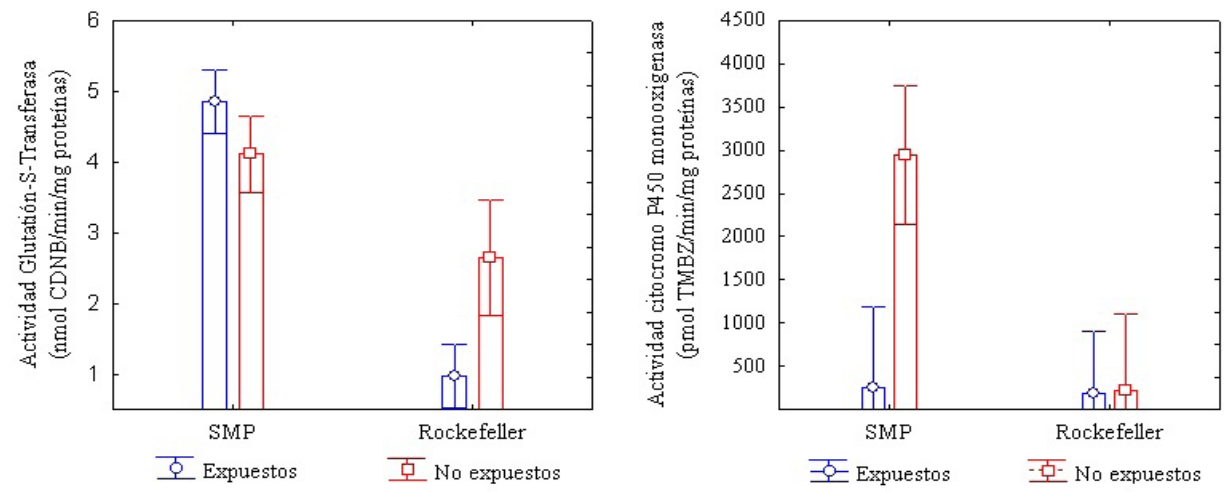

Figura 2. Actividad glutatión-S-transferasa (A) y actividad citocromo P450 monooxignasas (B) determinadas para cada cepa en el estadio de larva. Se realizó un test Mann Whitney (A: SMP ( $U=2005 ; \mathrm{p}=0,168)$; Rockefeller $(\mathrm{U}=307 ; \mathrm{p}=0.00001)$; B: SMP $(U=279 ; p=0,0000001)$; Rockefeller $(U=1824 ; p=0.04)$ utilizando el programa estadístico STATISTICA 7. Las barras representan los intervalos de confianza.

a sus respectivas $\mathrm{CL}_{90}$ del ATM, se encontró que la actividad fue más elevada en la cepa resistente que en la susceptible, tanto en los individuos expuestos, como en los no expuestos o controles. La actividad de las carboxilesterasas ( $\alpha$ y $\beta$ ) disminuyó en los sobrevivientes a la exposición a ATM en la cepa Miguel del Padrón 2011 con respecto al control. En la cepa Rockefeller disminuyó la actividad $\alpha$ carboxilesterasa, en los individuos expuestos, mientras que la actividad beta se mantuvo similar en los expuestos como en el control. La actividad de las GST se incrementó ligeramente en las larvas expuestas con respecto al control en la cepa Miguel del Padrón 2011, mientras que ocurrió lo contrario en la cepa Rockefeller. Por su parte, la mayor actividad de las MFO se encontró en los individuos control de la cepa resistente, destacándose una disminución posterior luego de ser expuestos. Valores similares se encontraron en la cepa susceptible. 


\section{Leyva-Silva et al.}

\section{DISCUSIÓN}

Determinar los mecanismos y niveles de resistencia en poblaciones de insectos expuestas a los insecticidas constituye un factor importante a tomar en cuenta para prolongar el empleo de cualquier método de control que sea efectivo contra insectos vectores de enfermedades al hombre.

Considerando que, en trabajos anteriores se relaciona la función de las enzimas detoxificadoras con la efectividad de la actividad insecticida de determinados aceites de plantas (35-37), se propuso que la resistencia a insecticida $y$, en particular, la resistencia metabólica encontrada en la cepa San Miguel del Padrón 2011, como una posible justificación del resultado encontrado anteriormente (25).

La cepa San Miguel del Padrón 2011 manifestó resistencia a los insecticidas evaluados en los estadios de larva, según los resultados obtenidos en este trabajo, y en el estadío adulto, según resultados obtenidos en estudios previos (25). Sin embargo, existen trabajos en los que solo se ha detectado resistencia en uno de los estadios. Montada et al, 2009 (38) al evaluar cipermetrina y lambdacialotrina en tres cepas de La Habana, solo encontró resistencia en el estado adulto. Los autores atribuyeron estos resultados a que los dos piretroides evaluados no eran insecticidas utilizados para el control larval. En contraste, en otros estudios se encontró que una cepa presionada con temefos por 7 años resultó ser resistente a deltametrina, tanto en el estadio larval como en el estadio adulto. Sin embargo, al evaluar esta cepa con lambdacialotrina solo se encontró resistencia en el estado adulto (39). La resistencia cruzada entre determinados insecticidas está fuertemente asociada al desarrollo de mecanismos de resistencia que median la protección a los insectos frente a varios compuestos (40). Aunque los insecticidas piretroides no son utilizados como larvicidas, la selección en el estado adulto con piretroides podría hacer que se potencie, en ambos estadios, mecanismos de resistencia que actúen frente a insecticidas a los que, inclusive, la población no ha sido expuesta (39).

Los niveles de resistencia encontrados con temefos en la cepa San Miguel del Padrón 2011, pudieran atribuirse a la presión de selección con este larvicida, aplicado durante más de 30 años en el programa de control vectorial cubano (38). Esto también pudo haber generado resistencia cruzada a lambdacialotrina, deltametrina y malatión en esta cepa, lo cual coincide con resultados encontrados por otros autores $(7,8,41,44)$.

Es de destacar la alta resistencia encontrada a bendiocarb, a pesar de haber sido usado eventualmente en tratamiento perifocal, para el control de Ae. aegypti en algunas provincias de Cuba (42). Sin embargo, en estudios realizados en Malasia se encontró resistencia frente a este compuesto, aún sin haberlo empleado con anterioridad en el control vectorial (43).

Una variedad de esterasas, oxidasas y un grupo de transferasas, median la detoxificación de aleloquímicos tóxicos en los insectos, mediante su transformación enzimática (44-46). Estos sistemas enzimáticos están implicados en la resistencia metabólica a carbamatos organofosforados, piretroides y DDT entre otros (26-28).

La implicación de los mecanismos de resistencia sobre la actividad biológica de los aceites es un tema que está siendo muy estudiado, cuando se valora la posibilidad de utilizar productos naturales para el control de insectos.

En nuestros resultados, los principales sistemas enzimáticos responsables de la resistencia metabólica en los insectos se encontraron sobreexpresados en esta cepa con respecto a la cepa susceptible. Aunque los valores de actividad de las carboxilesterasas fueron mayores en la cepa resistente respecto a la cepa Rockefeller, el valor medio disminuyó significativamente en las larvas expuestas a ATM respecto a las no expuestas o controles, en ambas cepas. Por otra parte, en cuanto a la actividad de las MFO, se encontró una disminución de la actividad en las larvas expuestas de ambas cepas, aunque el efecto fue

\section{Revista Biomédica}


Inhibición de enzimas detoxificadoras en Aedes aegypti

mayor en Rockefeller. Estos resultados evidencian la actividad inhibidora de ATM en la actividad esterasa y MFO.

Las enzimas esterasas y citocromo P450 monoxigenasas además de intervenir en la detoxificacion de los insecticidas sintéticos cumplen un rol importante en procesos como la reproducción, digestión y muda (47-48). Un desequilibrio en la funcionalidad de las enzimas mencionadas como consecuencia de la exposición al ATM pudiera justificar la actividad ovicida, larvicida, pupicida y teratogénica atribuida a este aceite (25).

No existe concordancia en los resultados obtenidos en trabajos similares con respecto al efecto de los aceites sobre la actividad de las enzimas detoxificadoras, lo cual pudiera estar relacionado al tipo de aceite empleado en cada caso particular. Cordeiro et al, 2013 (37) al enfrentar una cepa resistente a organofosforados y la cepa susceptible Rockefeller a WSMol (lectina soluble en agua obtenida de Moringa oleifera), encontró elevados niveles de $\alpha$-esterasas más que de $\beta$-esterasas para ambas cepas. La cepa susceptible presentó una estimulación de $\alpha$-esterasa mientras que en la cepa resistente no se evidenciaron cambios significativos para esta enzima. De manera similar, en otro estudio al evaluar la actividad de esterasas en larvas de Ae. aegypti expuestas a un extracto de Sapindus emarginatus, encontraron una reducción de la actividad $\beta$-esterasa, mientras que no encontró ninguna variación en la $\alpha$-esterasa (49). En nuestro estudio, la inhibición de las beta carboxilesterasas fue mayor que la de las alfa esterasas. Sin embargo, Joffe et al 2012 (50), encontró inhibición de la actividad de las citocromo P450 monooxigenasas, pero no de las esterasas al probar aceite de semilla de perejil y dillapiol en Musca domestica.

En contraste a la inhibición de las esterasas y MFO, el valor medio de actividad de las GST tuvo un ligero incremento en los individuos expuestos con respecto al control. Este resultado pudiera ser explicado por el hecho de que en los insectos las enzimas glutatión-S-transferasa no solo tienen como función mediar la detoxificación de xenobióticos, sino que también constituyen uno de los mecanismos protectores frente al estrés oxidativo que se genera por la exposición a tóxicos (51-52). Por lo tanto, el incremento de la actividad GST pudiera analizarse como un mecanismo protector y no como un mecanismo de resistencia frente al ATM. Sin embargo, el incremento de la actividad de las GST en larvas de Ae. aegypti resistentes expuestas a dos aceites, también se ha analizado como un mecanismo de detoxificación (53).

Los aceites, como los insecticidas, pueden ser detoxificados con la participación de diferentes enzimas. La exposición de los insectos a los aceites puede modificar la actividad o cantidad de las proteínas detoxificadoras ya sea aumentándola o disminuyéndola. En dependencia del efecto del aceite sobre la actividad detoxificadora puede ocurrir un desbalance hormonal, que se traduce en deterioro del crecimiento y finalmente la inducción de la muerte o la supervivencia del insecto (54-56).

El proceso de fotoisomerización de ATM rinde derivados oxigenados de verbenona y pulegona los cuales afectan el desarrollo fisiológico de Ae aegypti (25). Algunos autores, plantean que el efecto neurotóxico de los aceites esenciales y sus constituyentes se produce mediante la modulación de la actividad biológica de sus blancos moleculares, los cuales incluyen algunos canales iónicos (57) y a los receptores de tiramina, octopamina, $\mathrm{GABA}_{\mathrm{A}} \mathrm{y}$ acetilcolina $(46,58)$; otros autores plantean que, compuestos como timol, pulegona y carvacrol son moduladores alostéricos positivos de los receptores GABA en insectos (59). La presencia de mutaciones en los sitios blancos de la acción de los insecticidas es un mecanismo que media la resistencia a varios compuestos en diferentes especies de artrópodos (26). Este mecanismos pudiera ser la causa del incremento de la dosis de ATM que se encontró en trabajos previos (25).

De forma general, la actividad de las 
Leyva-Silva et al.

enzimas detoxificadoras disminuyó, lo que sugiere una baja probabilidad de manifestación de resistencia metabólica frente al ATM. Una variante de utilización de ATM sería su empleo en el control de poblaciones que no manifiesten este tipo de actividad enzimática (37) y, en poblaciones en las que se detecten niveles considerables de las GST, pudiera emplearse ATM combinado con sinergistas sintéticos para potenciar el efecto tóxico $(50,60-$ 61).

Los resultados de este trabajo y la demostrada actividad ovicida, larvicida, pupicida $\mathrm{y}$ teratogénica del aceite, avalan y permiten recomendar el uso del aceite de trementina modificado como un controlador de origen natural para cepas de Ae aegypti.

\section{REFERENCIAS}

1. WHO Impact of dengue. Global alert and response (GAR)2012a. http://www.who.int/csr/disease/dengue/ impact/en/index.html.

2. WHO Global strategy for dengue prevention and control 2012-2020. 2012b WHO, Geneva.

3. WHO Dengue and severe dengue. Factsheet no.117.2012c http://www.who.int/mediacentre / factsheets/fs117/en/.

4. Bisset JA, Rodriguez MM, Ricardo Y, Ranson H, Perez O, Moya M, et al. Temephos resistance and esterase activity in the mosquito Aedes aegypti in Havana, Cuba increased dramatically between 2006 and 2008. J Med Vet Entomol 2011;25: 233-9.

5. Ryan MF, Byrne O. Plant-insect coevolution and inhibition of acetylcholinesterase. J Chem Ecol 1988;14:1965-75

6. Wadley L, Stevers Ch, Bamford Goldeberg P, Berna F y Miller Christopher. Middle slone age bedding construction and settlement patterns of Sibudu South Africa. Sience 2011; 334(6061):1386-1391.

7. Tikar SN, Kumar A, Prasad GB, Prakash S. Temephos-induced resistance in Aedes aegypti and its cross-resistance studies to certain insecticides from India. Parasitol Research 2009;105(1):57-63.

8. Melo-Santos MAV, Varjal-Melo JJM, Araújo AP, Gomesa TCS, Paiva MHS, Regis LN, et al . Resistance to the organophosphate temephos: Mechanisms, evolution and reversion in an Aedes aegypti laboratory strain from Brazil. Acta Tropica 2010;113:180-189.

9. Rodríguez MM, Bisset JA, Pérez O, Montada D, Moya et al.Estado de la resistencia a insecticidas y sus mecanismos en Aedes aegypti en el municipio Boyeros. Rev Cubana Med Trop 2009;61(2): 187-198.

10. Rodríguez MM, Bisset JA, RicardoY, Pérez O, Montada D, Figueredo $\mathbf{D}$ et al. Resistencia a insecticidas organofosforados en Aedes aegypti (Diptera: Culicidae) de Santiago de Cuba, 1997-2009. Rev Cubana Med Trop 2010; 62(3):217-23.

11. Phasomkusolsil S, Soonwera M. Potential larvicidal and pupacidal activities of herbal essential oils against Culex quinquefasciatus Say and Anopheles minimus (Theobald).Southeast Asian J Trop Med Public Health 2010; 41(6):1342-51.

12. Chung IM, Song HK, Yeo MA, Moon HI. Composition and immunotoxicity activity of major essential oils from stems of Allium victorialis L. var. platyphyllum Makino against Aedes aegypti L. Immunopharmacol 2011; 33(3):480-83.

13. Hafeez F, Akram W, Shaalan EA. Mosquito larvicidal activity of citrus limonoids against Aedes albopictus. Parasitol Res. 2011; 109 (1):221-229.

14. Mathew J, Thoppil JE. Chemical composition and mosquito larvicidal activities of Salvia essential oils. Pharm Biol 2009; 49(5):456-463.

15. Pitarokili D, Michaelakis A, Koliopoulos G, Giatropoulos A, Tzakou O.Chemical composition, larvicidal evaluation, and adult repellency of endemic Greek Thymus essential oils against the mosquito vector of West Nile virus. Parasitol Res 2011; 109(2):425-430.

16. De Lima Santos ND, Santana K, Napoleao TH, Novais Santos G, Breitenbach LC,Do Amaral DM et al Oviposition stimulant and ovicidal activities of Moringa oleifera lectin on Aedes aegypti Plos One 2013: 7(9): e44840.doi:10.1371/journal.pone.0044840.

17. Tong $\mathbf{F}$, Bloomquist $\mathbf{J}$ Plant essential oils affect toxicities of carbaryl and permethrin against Aedes egypti (Diptera : Culicidae). J Med Entomol 2013; 50 (4):826-832.

18. Noleto Dias C, Fernandes D.Essential oils and their compounds as Aedes aegypti L. (Diptera: Culicidae) larvicides: review Parasitol Res 2013 DOI 10.1007/ s00436-013-3687-6

19. Roig JT. Diccionario botánico de nombres vulgares cubanos. Tercera Edición Editora del Consejo Nacional de Universidades, La Habana, Cuba. 1965:1142pp

20. Pino O, Sánchez Y, Rodríguez H, Correa TM, Demedio J, Sanabria JL. Caracterización química y actividad acaricida del aceite esencial de Piper aduncum subsp. ossanum frente a Varroa destructor. Prot Vegetal 2011; 26 (1):

21. Pino O, Sánchez Y, Rojas MM, Rodríguez H, Abreu $\mathbf{Y}$ et al, Composición química y actividad plaguicida del aceite esencial de Melaleuca quinquenervia (Cav) 
Inhibición de enzimas detoxificadoras en Aedes aegypti

S.T. Blake . Prot Vegetal 2011; 26(3)

22. Leyva M, Marquetti MC, Tacoronte JÁ, Tiomno O, Montada D. Efecto inhibidor del aceite de trementina sobre el desarrollo de larvas de Aedes aegypti (Diptera: Culicidae) Rev Cubana Med Trop 2010;62(3):212-16.

23. Leyva M, Tiomno O, Tacoronte JE, Marquetti MC, Montada D. Essential Plant Oils and Insecticidal Activity in Culex quinquefasciatus 2012 Insecticides Pest Engineering ISBN 978-953-307-895-3 Ed Intech. 221-238pp

24. Leyva M, Castex M, Montada D, Quintana D, Lezcano D, et al. Actividad repelente de formulaciones del aceite esencial de Melaleuca quinquenervia (Cav.) S.T. Blake (Myrtales:Myrtaceae) en mosquitos. Anales de Biología 2012; 34: 47-56.

25. Leyva M, Marquetti MC, French L, Montada D,Tiomno $\mathbf{O}$,Tacoronte JE. Efecto de un aceite de trementina obtenido de Pinus tropicalis Morelet 1851 sobre la biología de una cepa de Aedes (Stegomyia) aegypti Linnaeus 1762 resistente a insecticidas. Anales de Biología 2013;35: 75-88.

26. Hemingway $\mathbf{J} \mathbf{H}$, Mccarroll $\mathbf{L}$, Ranson $\mathbf{R}$. The molecular basis of insecticide resistance in mosquitoes. Insect Bioch Molecular Biology 2004:34: 653-665.

27. Li X, Schuler M, Berenbaum MR. Molecular mechanisms of metabolic resistance to synthetic and natural xenobiotics. Annu Rev Entomol 2007; 52:23153.

28. Ranson H, Hemingway J. Insect pharmacology and control: glutathione S-transferases, Oxford, 2004 UK, Elsevier.

29. OMS. Instrucciones para determinar la susceptibilidad o resistencia a insecticidas en larvas de mosquito. WHO/VBC/81.807

30. Mazarri MB, Georghiou GP. Characterization of resistance to orgaphosphate, carbamate and pyrethroid insecticides in field populations of Aedes aegypti from Venezuela. J Am Mosq Control Assoc 1995; 11:315-22.

31. Dhiman S, Rabha B, Yadav K, Baruah I, Veer V .Insecticide susceptibility and dengue vector status of wild Stegomyia albopicta in a strategically important area of Assam, India. Parasit Vectors 2014; 7: 295. Published online Jul 1, 2014.

32. Brogdon WG, Mcallister JC. Insecticide resistance and vector control. Emerg Infect Dis. 1998;4, 605-13.

33. French L, Rodríguez MM, Bisset JA, Ricardo Y, Gutiérrez G, Fuentes I. Actividad incrementada de las enzimas citocromo P450 monooxigenasas en cepas cubanas de Aedes aegypti de referencia, resistentes a insecticidas. Rev Cubana Med Trop 2013; 65(3): 328-338.

34. Rodríguez M, Bisset J, Díaz C, Soca A. Adaptación de los métodos en placas de microtitulación para la cuantificación de la actividad de esterasas y glutations-transferasa en Aedes aegypti. Rev Cubana Med Trop 2001;53(1): 32-6.

35. Larson RT, Lorch JM, Pridgeon JW, Becnel JJ, Clarck GG, Lan Q. The biological activity of $\alpha$-mangostin, a larvicidal botanic mosquito sterol carrier protein-2 inhibitor. J Med Entomol 2010; 47:249-257.

36. Tripathy A, Samanta L, Sachidananda D, Parida S, Marai $\mathbf{N}$ et al. The mosquitocidal activity of methanolic extracts of Lantana camara Root and anacardium occidentalle leaf: role of GST in insecticidal resistance. J Med Entomology 2011; 48(2):291-295.

37. Cordeiro Agra-Neto A, Henrique Napoleão T, Viana Pontual E, Diniz de Lima Santos N, Andrade Luz L, Fontes de Oliveira CM, et al. Effect of Moringa oleifera lectins on survival and enzyme activities of Aedes aegypti larvae susceptible and resistant to organophosphate. Parasitol Res 2013 DOI 10.1007/ s00436-013-3640-8.

38. Montada D, Leyva M, Silva Y, Marquetti MC. Susceptibilidad de 3 cepas de Aedes aegypti asociada con la aplicación de 3 insecticidas. Rev Cubana Med Trop 2009;61(2):199-209.

39. French L. Estudio de la resistencia a insecticidas en los estadios de larva y adulto de Aedes aegypti (Diptera: Culicidae) Tesis para optar por el grado de Master en Entomología medica y Control de Vectores 2012. Instituto de Medicina Tropical "Pedro Kourí" Cuba.

40. Hemingway J, Georghiou GP. Studies on the acetylcholinesterase of Anopheles albimanus resistant and susceptible to organophosphate and carbamate insecticides. Pestic Biochem Physiol 1983; 19:167-171.

41. Rodriguez M. Estudio de la resistencia a insecticidas. Tesis doctoral. Instituto de Medicina Tropical Pedro Kouri 2008. 114 p

42. Castex M, Montada D, González I, Estevez S, San Blas O, González R. Efectividad del tratamiento residual (Perifocal) con Ficam (Bendiocarb) 80 WP en el control del mosquito Aedes aegypti en el Área de Salud XX Aniversario A, en Santa Clara. Cuba. Rev Cubana Med Trop 2008; 60(1):61 -62.

43. Seau-Rong L, Tan-Wei-Ann A, Wasi-Ahmad N, Ham-Lim L, Sofian-Azirum M. Insecticidal susceptibility status of field collected Aedes (Stegomya) aegypti (L) at dengue endemic site in Sham Alam Selengor Malasya . Southeast Asian J Tropical Med Public Health 2012;43(1):34-47.

44. Schuler MA. The role of cytochrome 450 monooxygenases in plant insect interactions. Plant Physiology 1996;112: 1411-19.

45. Ortego F, Lopez-Olguin JF, Ruiz M, Castanera P. 


\section{Leyva-Silva et al.}

Effect of toxic and deterrent terpenoids on digestive proteases and detoxication enzyme activities of Colarado potato beetle larvae. Pest Biochem Physiol 1999; 63: 76-84.

46. Rattan RS. Mechanism of action of insecticidal secondary metabolites of plant origin. Crop Protection 2010:29 913-20.

47. Lassiter MT, Apperson CS, Roe RM. Juvenile hormone metabolism during the fourth stadium and pupal stage of the southern house mosquitoes Culex quinquefasciatus Say. J Insect Physiol 1995; 41: 869876.

48. Shanmugavelu M, Baytan AR,Chesnut SJ,Bonning BC. A novel protein that bindsjuvenile hormone esterase in fat body tissues and pericardial cells of the tobacco horn worm Manduca sexta L . J Biol Chem 2000; 275 :1802-06.

49. Koodalingam A, Mullainadhan P, Arumugam M. Effects of extract of soapnut Sapindus emarginatus on esterases and phosphatases of the vector mosquito Aedes aegypti (Diptera Culicidae). Acta Tropica 2011;118: 27-36.

50. Joffe T, Gunning RV, Allen GR, Kristensen M, Alptekin S, Field LM et al. Investigating the potential of selected natural compounds to increase the potency of pyrethrum against houseflies Musca domestica (Diptera: Muscidae). Pest Manag Sci 2012, 68:178-184.

51. Vontas JG, Small GJ, Hemingway J. Glutathione S-transferases as antioxidant defence agents confer pyrethroid resistance in Nilaparvata lugens. Biochem J 2001;357: 65-72.

52. Zelck UE, Janowsky V B. Antioxidant enzymes in intramolluscan Schistosoma mansoni and ROS-induced changes in expression. Parasitology 2004; 128: 493501.

53. Tripathy A, Samanta L, Sachidananda D, Parida S, Marai n, Hazra RK, et al .The mosquitocidal activity of methanolic extracts of Lantana camara Root and Anacardium occidentalle leaf: role of GST in insecticidal resistance. J Med Entomol 2011;48(2):291295.

54. Macedo MLR, Freire MGM, Silva MBR, Coelho LCBB. Insecticidal action of Bauhinia monandra leaf lectin (BmoLL) against Anagasta kuehniella (Lepidoptera: Pyralidae), Zabrotes subfasciatus, and Callosobruchus maculatus (Coleoptera: Bruchidae). Comp Biochem Physiol A 2007; 146:486-498

55. Babu SR, Subrahmanyam B. Bio-potency of serine proteinase inhibitors from Acacia senegal seeds on digestive proteinases, larval growth, and development of Helicoverpa armigera (Hübner). Pest Biochem Physiol 2010; 98:349-358.

56. Napoleão TH, Pontual EV, Lima TA, Santos NDL, Sá RA, Coelho LCBB, et al. Effect of Myracrodruon urundeuva leaf lectin on survival and digestive enzymes of Aedes aegypti larvae. Parasitol Res 2012;110:609616.

57. IRAC 2012 Web site at www.irac-online.org. visited date March5th, 2012

58. Blenau W, Rademacher E, Baumann A. Plant essential oils and formamidines as insecticides/ acaricides: what are the molecular targets?. Apidologie 2012, 43:334-47.

59. Tong F, Coats JR. Effects of monoterpenoid insecticides on $[3 \mathrm{H}]-\mathrm{TBOB}$ binding in house fly GABA receptor and 36Cl- uptake in American cockroach ventral nerve cord. Pest Biochem. Physiol 2010;98, 317-324.

60. Yadav S, Mittal PK, Saxena PN, Singh RK. Effect of synergist piperonyl butoxide (PBO) on the toxicity of some essential oils against mosquito larvae. J Commun Dis 2009; 41:33-38.

61. Waliwitiya R, Nicholson RA, Kennedy CJ, Lowenberger CA The synergistic effects of insecticidal essential oils and piperonyl butoxide on biotransformational enzyme activities in Aedes aegypti (Diptera: Culicidae). J Med Entomol 2012; 49:614-623. 